\title{
Probability Distribution Fitting and Correlation Analysis of Engineering Education Quality Assessment
}

\author{
Juan Chen ${ }^{\mathrm{a}}$, Bin Duan ${ }^{\mathrm{b}}$ and Xiangshuai $\mathrm{Qu}^{\mathrm{c}}$ \\ Xiangtan University, Xiangtan 410005 , China. \\ achenjuanxtu@163.com, bdb61850@163.com, ' quxiangshuai@163.com
}

Keywords: Quality assessment; Engineering Education; Probability distribution fitting; Correlation Analysis.

\begin{abstract}
With the development of the engineering education, quality assessment has received widespread attention. In China, universities adopt deterministic analysis on quality assessment, which regards course exam scores as the assessment metrics and there are very few other assessment models that may cause the inaccuracy of assessment results. To solve this problem, the paper puts forward engineering education quality assessment decision-making system containing exam assessment model, S-ISAL(Space-based Information Search and Analysis Learning) assessment model and third-party assessment model. Using @risk simulation tool, the probability distribution of the three assessments is fitted according to sample data, and then analyzing the correlation among these assessments to get the probability distribution of quality assessments. The probability distribution provides scientific information for decision-making and planning in education management.
\end{abstract}

\section{Introduction}

Different from the Sydney accord and the Dublin accord, the updated Washington regard the "complex engineering problem" as the background, the student as the center and insist on outcome based education (OBE), to ensure continuous improvement of the quality [1]. Engineering education quality assessment has become the basic idea of the engineering education accreditation. Determining the training objectives, analyzing how to reach the training objectives, confirming how well does it achieves the object, the quality assessment is highlighted by the OBE philosophy, and how to do the assessment has become an important issue [2].

In China, engineering education quality assessment is mainly supported by the course assessment [3]. One concern of quality assessment is that only take the average score as the standard, which did not take the influence that the difficulty on the test brings to the exam assessment into consideration and there are very few assessment models, lack of comprehensiveness. The other concern is the current quality assessment is based on the existing scores and cannot be used for education management planning and decision making [4].

In order to improve the scientificalness of the quality assessment, the paper presents a decision-making system for engineering education quality assessment that including exam assessment model, S-ISAL assessment model, third-party assessment model[5]. Through probability distribution fitting and the correlation analysis, the latter two assessment model can help adjust influence of exam difficulty, at the same time; these assessment models cooperatively decided the final quality assessment results [6]. The probability distribution of the quality assessment also can provide scientific and rational decision-making in education management.

\section{Engineering Education Quality Assessment Decision-making System}

This paper puts forward decision-making system for engineering education quality assessment is as shown in Fig. 1. 


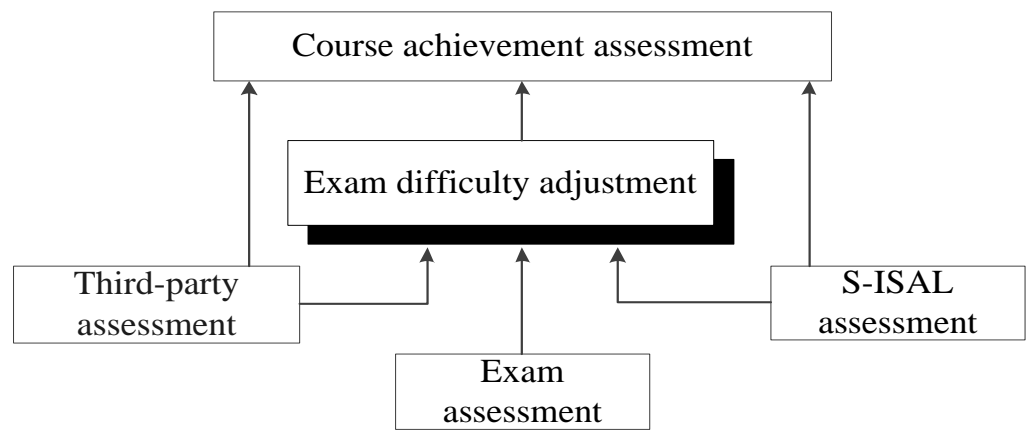

Fig. 1 Diagram of engineering education quality assessment decision-making system

Exam assessment: the assessment model regards the student scores as the assessment metrics. The higher score, the better score assessment result.

S-ISAL assessment: The assessment model is based on information searching of space and analysis of the study, teachers' teaching and students' learning process will leave evidence in space. We can form S-ISAL assessment model, by combining process evaluation with result evaluation, diagnostic evaluation with formative assessment, and surround the core of the course achievement assessment to cultivate students' comprehensive ability in information searching, analysis expression, and team cooperation and so on. The assessment model with the idea of task driving plays an auxiliary role in quality assessment.

Third-party assessment: The assessment model is mainly through third-party organizations (employer, recruitment website and consulting company, etc.) to evaluate the students' ability to graduate which reflect the concrete work.

This quality assessment decision-making system improved and perfected the current exam assessment model comprehensively, which took the influence that the difficulty on the exam brings to the examination assessment into consideration [7.8].

\section{Probability Distribution Fitting of Quality Assessment}

The current engineering education quality assessment is based on the existing scores, which cannot be used for in planning and decision-making of education management. There are two analysis methods of education planning decision-making: deterministic analysis and stochastic analysis [9].

Deterministic analysis: The deterministic analysis adopts a single value to make decisions, but the decision provides only one outcome, although it may look like this, actually, the average way of thinking that lack of correlation analysis may lead to mistakes in decision-making.

Stochastic analysis: all possible outcomes are considered and the probabilities of that are also been calculated. Then, making decisions according to the probability distribution of the decision variables, and obtaining the probability distribution of the decision objectives. The analysis provides scientific and rational decision-making information, for example: the variation range of decision results, the driving factors that have influence on decision results, etc.

\subsection{Data Collection}

Obtain the sample data respectively, namely: exam scores, data of third-party assessment and S-ISAL teaching scores. As shown in Table 1, 2, 3.

Table 1 sample data of exam assessment

\begin{tabular}{|c|c|c|c|c|c|c|c|c|c|c|c|c|c|c|}
\hline 79 & 70 & 76.5 & 78 & 79.5 & 77.5 & 77.5 & 80.5 & 78.5 & 80 & 80.5 & 80 & 78 & 78 & 74.5 \\
\hline 81 & 78.5 & 80 & 79 & 81 & 74.5 & 80 & 80.5 & 81.5 & 80 & 79.5 & 80.5 & 79 & 79 & 79.5 \\
\hline
\end{tabular}

Table 2 sample data of third-party assessment

\begin{tabular}{|l|l|l|l|l|l|l|l|l|l|l|l|l|l|l|}
\hline 92 & 91 & 93 & 82.5 & 50.5 & 91.5 & 69.5 & 69.5 & 46 & 73 & 93 & 93.5 & 93.5 & 71 & 96.5 \\
\hline 82 & 92.5 & 75 & 74 & 87 & 81 & 94.5 & 82 & 88 & 97 & 75 & 62 & 72.5 & 77.5 & 76 \\
\hline
\end{tabular}


Table 3 sample data of S-ISAL assessment

\begin{tabular}{|l|l|l|l|l|l|l|l|l|l|l|l|l|l|l|}
\hline 79 & 70 & 76.5 & 78 & 79.5 & 77.5 & 77 & 80 & 78.5 & 80 & 80.5 & 80 & 78 & 78 & 74.5 \\
\hline 81 & 78.5 & 80 & 79 & 81 & 74.5 & 80 & 80 & 81.5 & 80 & 79.5 & 80.5 & 79 & 79 & 79.5 \\
\hline
\end{tabular}

\subsection{Probability Distribution Fitting}

Using @risk software to simulate probability distribution of the three assessment models and get the probability density functions. Distribution fitting results are shown in Fig 2.3.4.

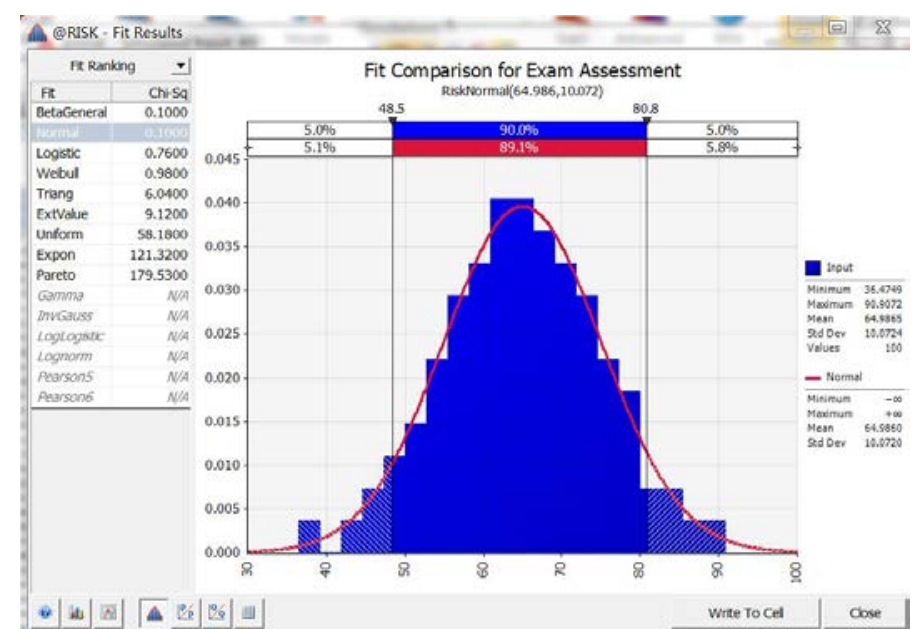

Fig. 2 Fitting results of exam assessment

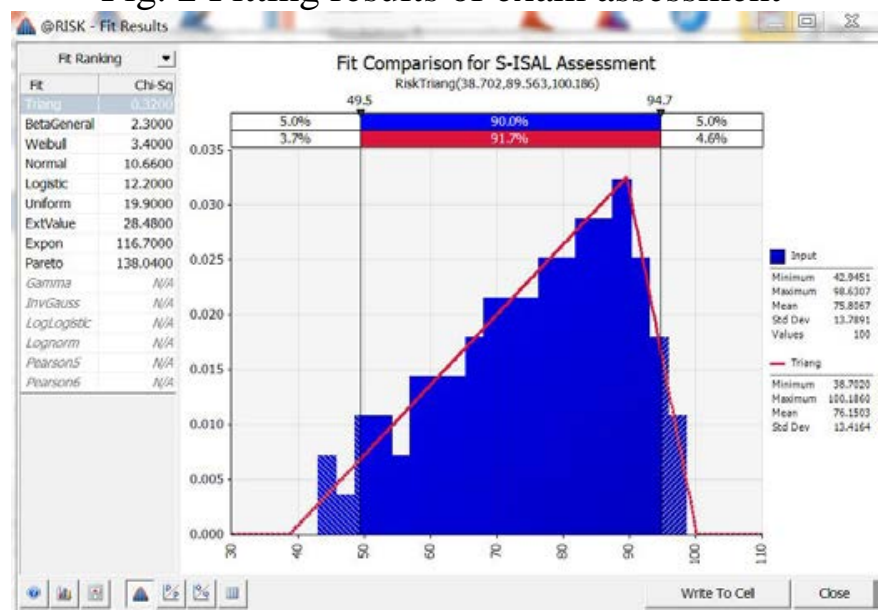

Fig. 3 Fitting results of S-ISAL assessment

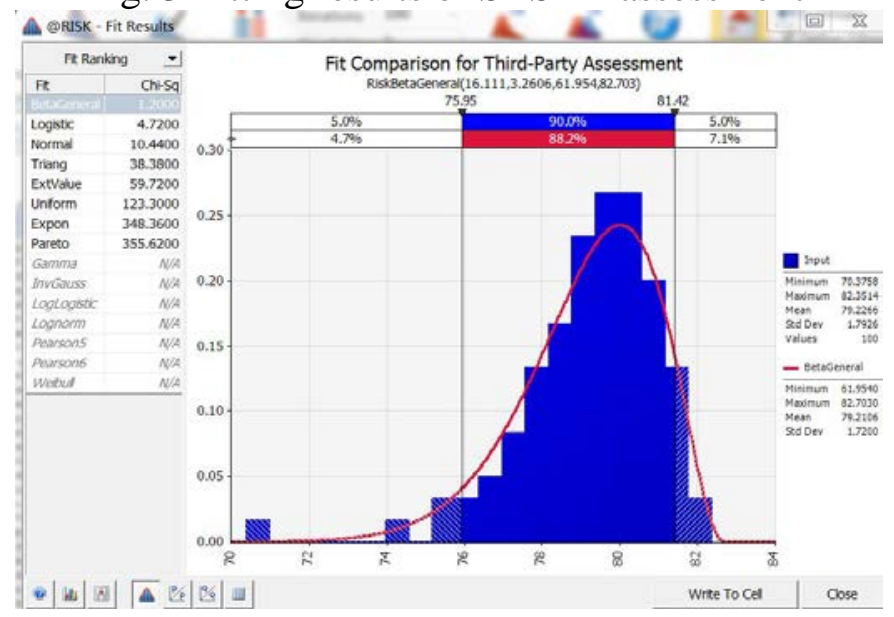

Fig.4 Fitting results of third-party assessment

The exam assessment obeys Normal distribution $(64.97,9.90)$ and the mean is 64.9765 , the Std Dev (Standard Deviation) is 10.0724. S-ISAL assessment obeys Triang distribution (36.47, 88. 96, 100.96) and the mean is 76.1503, the Std Dev (Standard Deviation) is 13.4164. Third-party 
assessment obeys Weibull distribution $(59.54$, 80.32) with the mean 79.2266 and Std Dev (Standard Deviation) 1.7926.

Compare the fitting results in different weights on the three assessments, and then select the most appropriate weight configuration to get scientific and reasonable probability distribution of quality assessment.

The paper takes average weights and calculates the average fitting result of the three assessments by the following equation.

Fitting result $=\frac{\text { Exam assessment }+S-I S A L \text { assessment }+ \text { Third-party assessment }}{3}$

According to the equation (1), the final result is as show in fig5.

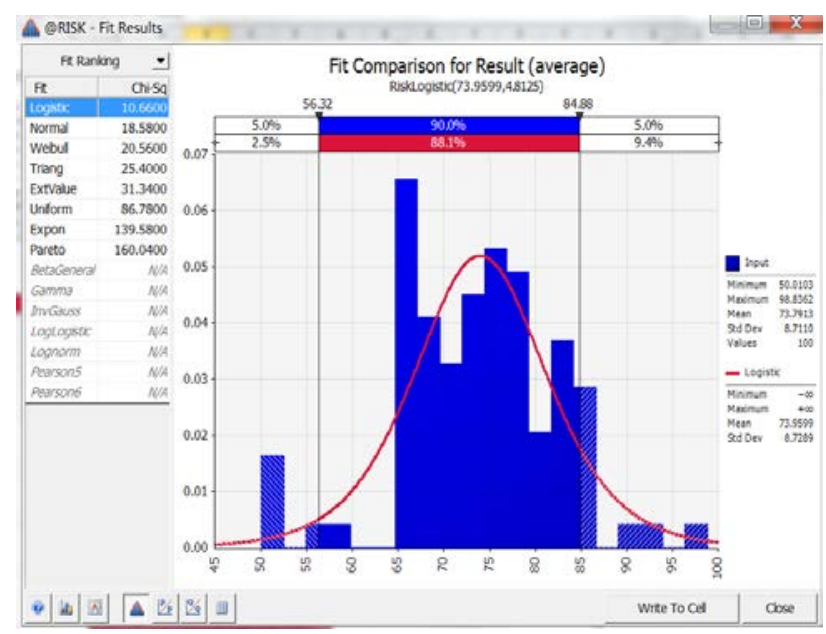

Fig. 5 Fitting results of quality assessment

The achievement assessment obeys Logistic distribution and the mean is 73.7913, the Std Dev(Standard Deviation) is 8.7110. Quality Assessment is determined by equation (2).

Assessment $=\frac{\text { Average result }}{\text { target value }}$

From the equation (2), we can get the assessment is 0.737 (the target value is 100).

\section{Correlation Analysis}

Using the @risk software to analysis Correlation among the three assessment model to help adjust the influence that the difficulty effects on the exam assessment. Correlation is as show in fig 6.

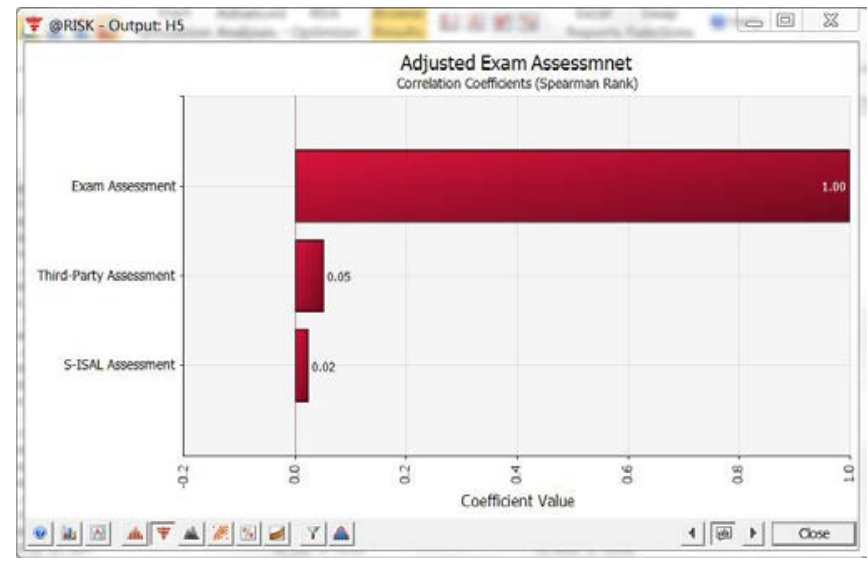

Fig .6 Correlation analyses among the three assessment model

From the fig 5, get the final adjustment equation as equation 3.

Adjusted exam assessment $=$ Exam assessment $+0.05 *$ Third - party assessment

$$
+0.02 * \mathrm{~S}-\mathrm{ISAL} \text { assessment }
$$


The distribution of adjusted exam assessment is as show in fig 7, that obeys the Logistic with the mean70.3637, Std Dev (Standard Deviation) 10.1303.

The comparison between Fig 1 and Fig 7 shows that the mean of the exam assessment being increased, which weakens the influence on the difficulty.

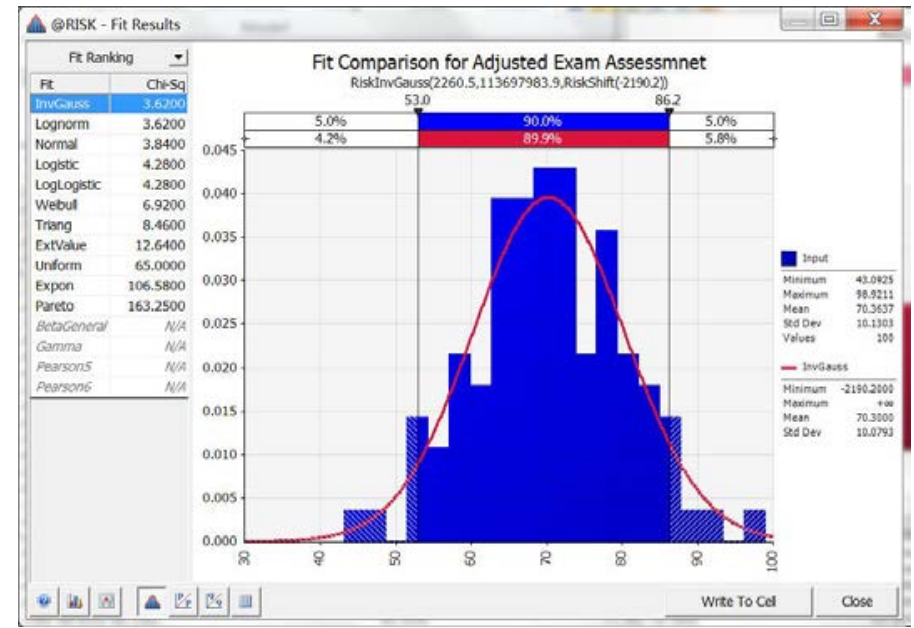

Fig. 7 Fitting results of adjusted exam assessment

According the steps that described in section 3, get the fitting results of final achievement assessment as show in fig8, and calculate the final assessment is 0.7527

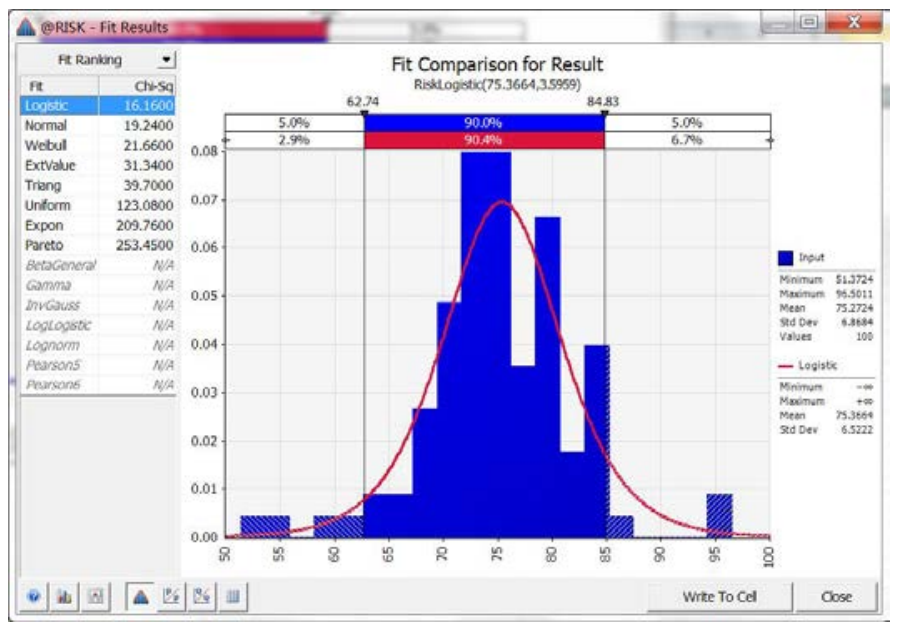

Fig. 8 Fitting results of final achievement assessment

\section{Conclusion}

Engineering Education Accreditation developed rapidly in recent years. In China, universities adopt the average score of the sample as the assessment metrics without considering the correlation while there is a doubt on the accuracy of assessment results. To solve this problem, the paper put forward engineering education quality assessment decision-making system using stochastic analysis that can get the scientific and accurate assessment results.

\section{Acknowledgment}

The research is supported by Regular Institutions of Higher Education Teaching Reform Project of Hunan Province and Regular Institutions of Higher Education Information Teaching Application Projects of Hunan Province. The authors would like to show great thanks for these support. 


\section{References}

[1]. Subbarao, E.C. "India’s Higher Engineering Education: Opportunities and Tough Choices," Current Science, 104, 55-66, 2013J. Clerk Maxwell, A Treatise on Electricity and Magnetism, 3rd ed., vol. 2. Oxford: Clarendon, 1892, pp.68-73.

[2]. D. Bowater, "Quality Assurance is Brazil's main concern," Times Higher Education, pp. 18, 2014.

[3]. M. M. U. Faiz, U. Mansoor, S. M. Asad, and K. Mahmood, "Using faculty course assessment report for the assessment of an associate degree course in engineering technology program," in Engineering Education, 2014 IEEE 6th International Conference on, 2014.R. Nicole, "Title of paper with only first word capitalized,” J. Name Stand. Abbrev. In press.

[4]. P.G. Altbach and R.M. Bassett, "Nix the BRICs - At least for the Higher Education Debate," International Higher Education, vol. 77, pp. 2-5, $2014 .$.

[5]. R.H.L. Pedrosa, "The Research University in Brazil: 1930 and 2030," International Higher Education, vol. 77, pp. 16-17, 2014.

[6]. J. Knight, "Building a Regional Academic Credit System in Latin America, "International higher Education, no. 44, pp. 18-20, 2015.

[7]. M. Souto-Otero, J. Huisman, M. Beerkens, H. de Wit and S. Vujić, "Barriers to international student mobility: Evidence from the ERASMUS program", Educational Researcher, pp. 70-77, 2013.

[8]. Reimers, J.E., Farmer, C.L. and Klein-Gardner, S.S. "An Introduction to the Standards for Preparation and Professional Development for Teachers of Engineering," Journal of Pre-College Education Research, 5, 40-60, 2015.

[9]. Moore, T.J., Glancy, A.W., Rank, K.M., Kersten, J.A., Smith, K.A. and Stohlmann, “M.S. A Framework for Quality K-12 Engineering Education,” Research and Development. Journal of Pre-College Engineering Education Research, 4, 1-13, 2014. 